\title{
Facile Group IV Based C-H Bond Activation: Confirmation of Classic Benzyne Mechanism by DFT. Extension to Adamantyl Activation.
}

\author{
Motasem Suleiman ${ }^{1}$, Fioralba Taullaj ${ }^{2}$, and Ulrich Fekl ${ }^{2}$ \\ ${ }^{1}$ University of Toronto \\ ${ }^{2}$ University of Toronto - Mississauga
}

October 1, 2020

\begin{abstract}
Group IV organometallic complexes are promising systems for $\mathrm{C}-\mathrm{H}$ bond activation. We are interested in the C-H bond activation of the $\mathrm{CH} 2$ positions of the adamantyl group, since these positions are particularly hard to activate and to functionalize. As a potential platform for activation of that important alkyl group, we consider the alkyl bonded to the cyclopentadienyl in a substituted bis-cyclopentadienyl group IV metal diphenyl complex. The mechanism proposed in the classic paper reporting such activation using $\mathrm{Zr}(\mathrm{IV})$ (Erker and Mühlenbernd, 1987) involves an $\eta 2$-benzyne complex intermediate. This current work reports a computational analysis of the problem through Density Functional Theory (DFT). We found that the two-step mechanism proposed for activation of $\mathrm{C}(\mathrm{Me}) 2-\mathrm{Ph}$ or tert- $\mathrm{Bu}$ groups using $\mathrm{Zr}(\mathrm{IV})$ is indeed confirmed by DFT and that it can be extended to Ti and Hf. We further found that the system can be successfully extended to the adamantyl group. The first step involves formation of the benzyne complex, which can also be described as a metallacyclopropene. In the second step, the cyclopentadienyl-bound alkyl is activated in the coordination sphere of the metal via proton transfer to the bound benzyne, which, if the metallacyclopropene description is chosen, resembles a $\sigma$-bond metathesis. The C-H bond activation of adamantyl through this approach is thermodynamically and kinetically feasible. Selective $\alpha$-CH bond activation should be achievable with $\mathrm{Ti}$ (under thermodynamic control), and selective $\gamma$-CH bond activation with $\mathrm{Zr}$ (under kinetic or thermodynamic control).
\end{abstract}

\section{Hosted file}

MS_Manuscript_Sept30.pdf available at https://authorea.com/users/363679/articles/484366facile-group-iv-based-c-h-bond-activation-confirmation-of-classic-benzyne-mechanism-bydft-extension-to-adamantyl-activation 